University of Nebraska - Lincoln

DigitalCommons@University of Nebraska - Lincoln

$11-2006$

\title{
Comments on "On the Distribution of the Product of Independent Rayleigh Random Variables"
}

\author{
Saralees Nadarajah \\ University of Nebraska-Lincoln, saralees.nadarajah@manchester.ac.uk \\ Samuel Kotz \\ George Washington University
}

Follow this and additional works at: https://digitalcommons.unl.edu/statisticsfacpub

Part of the Statistics and Probability Commons

Nadarajah, Saralees and Kotz, Samuel, "Comments on "On the Distribution of the Product of Independent Rayleigh Random Variables"'" (2006). Faculty Publications, Department of Statistics. 3.

https://digitalcommons.unl.edu/statisticsfacpub/3

This Article is brought to you for free and open access by the Statistics, Department of at DigitalCommons@University of Nebraska - Lincoln. It has been accepted for inclusion in Faculty Publications, Department of Statistics by an authorized administrator of DigitalCommons@University of Nebraska - Lincoln. 


\section{Comments and Replies}

\section{Comments on "On the Distribution of the Product of Independent Rayleigh Random Variables"}

Saralees Nadarajah and Samuel Kotz

\begin{abstract}
It is pointed out that the result in Salo et al. "The Distribution of the Product of Independent Rayleigh Random Variables" IEEE Trans. Antennas Propag., vol. 54, pp. 639-643, Feb. 2006, is a particular case of a much more general result known since the 1970s. A general technique (known as the $\boldsymbol{H}$ function technique) is described that can be used derive a wide range of results similar to Salo et al.
\end{abstract}

Index Terms-Fading channels, generalized gamma distribution, Rayleigh distribution.

\section{INTRODUCTION}

The recent paper by Salo et al. [1], derived the exact distribution of a product of $n$ independent Rayleigh random variables. In particular, [1] considered the distribution of the product

$$
Y=\prod_{i=1}^{n} X_{i}
$$

where $X_{i}, i=1,2, \ldots, n$ are independent Rayleigh random variables with the pdf

$$
f_{X_{i}}(x)=\frac{x}{\sigma_{i}^{2}} \exp \left(-\frac{x}{2 \sigma_{i}^{2}}\right)
$$

for $x>0$. The paper [1] derived expressions for the pdf and the cumulative distribution function (cdf) of $Y$, see [1, Eqs. (8)-(15)].

In this note, we would like to point out that the results on the pdf of $Y$ given by [1] has been known before. Section II describes a much more general result derived by Professor Mathai [2] in the 1970s. In Section III, we describe a general technique known as the $H$-function technique which can be used to derive a wide range of results similar to and containing [1] and [2].

\section{Mathai’s Results}

Consider the random variable $Z$ defined by

$$
Z=\frac{\prod_{i=1}^{m} X_{i}}{\prod_{i=m+1}^{N} X_{i}}
$$

where $X_{i}, i=1,2, \ldots, N$ are independent generalized gamma random variables with the pdf

$$
f_{X_{i}}(x)=\frac{\beta_{i} a_{i}^{\alpha_{i} / \beta_{i}} x^{\alpha_{i}-1}}{\Gamma\left(\alpha_{i} / \beta_{i}\right)} \exp \left(-a_{i} x^{\beta_{i}}\right)
$$

Manuscript received March 1, 2006; revised April 14, 2006.

S. Nadarajah was with the University of Nebraska, Lincoln, NE 68583 USA. $\mathrm{He}$ is now with the School of Mathematics, University of Manchester, Manchester M60 1QD, U.K. (e-mail: saralees.nadarajah@manchester.ac.uk).

S. Kotz is with the George Washington University, Washington, DC 20052 USA.

Digital Object Identifier 10.1109/TAP.2006.884313 for $x>0$. Note that (1) is a particular case of (2) for $m=N, \alpha_{i}=2$, and $\beta_{i}=2$. Reference [2] derived expressions for the moments and the pdf of $Z$, showing that

$$
\begin{aligned}
E\left(Z^{h}\right)= & C \prod_{i=1}^{m}\left\{a_{i}^{-h / \beta_{i}} \Gamma\left(\frac{\alpha_{i}+h}{\beta_{i}}\right)\right\} \\
& \times \prod_{i=m+1}^{N}\left\{a_{i}^{h / \beta_{i}} \Gamma\left(\frac{\alpha_{i}-h}{\beta_{i}}\right)\right\}
\end{aligned}
$$

and

$$
f_{Z}(z)=C \frac{1}{z} H_{n, m}^{m, n}\left(\begin{array}{l|l}
\frac{a z}{a^{\prime}} & \begin{array}{l}
\left.1-\frac{\alpha_{m+1}}{\beta_{m+1}}, \frac{1}{\beta_{m+1}}\right), \\
\ldots,\left(1-\frac{\alpha_{N}}{\beta_{N}}, \frac{1}{\beta_{N}}\right) \\
\left(\frac{\alpha_{1}}{\beta_{1}}, \frac{1}{\beta_{1}}\right), \\
\ldots,\left(\frac{\alpha_{m}}{\beta_{m}}, \frac{1}{\beta_{m}}\right.
\end{array}
\end{array}\right)
$$

for $z>0$, where

$$
\begin{aligned}
& C=\left\{\prod_{i=1}^{N} \Gamma\left(\frac{\alpha_{i}}{\beta_{i}}\right)\right\}^{-1} \\
& n=N-m \\
& a=\prod_{i=1}^{m} a_{i}^{1 / \beta_{i}}
\end{aligned}
$$

and

$$
a^{\prime}=\prod_{i=m+1}^{N} a_{i}^{1 / \beta_{i}} .
$$

For a definition of the $H$-function see [3] and [4]. Reference [2] also considered two particular cases of (3). In the case $\beta_{1}=\beta_{2}=\cdots=$ $\beta_{N}=\beta$, [2] showed that (3) can be reduced to

$$
f_{Z}(z)=C \frac{\beta}{z} G_{n, m}^{m, n}\left(\frac{a_{1} \cdots a_{m} z^{\beta}}{a_{m+1} \cdots a_{N}} \mid \begin{array}{l}
1-\frac{\alpha_{i}}{\beta}, \\
i=m+1, \ldots, N \\
\frac{\alpha_{i}}{\beta}, \ldots, \ldots, m \\
i=1, \ldots, m
\end{array}\right)
$$

for $z>0$. In the case, where $\beta_{i}$ are rational numbers, one can find a $\beta$ such that $1 / \beta_{i}=m_{i} / \beta, i=1,2, \ldots, N$ (where $m_{1}, m_{2}, \ldots, m_{k}$ are positive integers) and [2] showed that (3) can be reduced to

$$
f_{Z}(z)=C \frac{\beta}{z} G_{n^{\prime}, m^{\prime}}^{m^{\prime}, n^{\prime}}\left(t z^{\beta}\left(\begin{array}{l}
1-\frac{\alpha_{i}}{\beta}-\frac{r}{m_{i}}, \\
r=0,1, \ldots, m_{i}-1, \\
i=m+1, m+2, \ldots, N \\
\frac{\alpha_{i}}{\beta}+\frac{r}{m_{i}}, \\
r=0,1, \ldots, m_{i}-1, \\
i=1,2, \ldots, m
\end{array}\right)\right.
$$

for $z>0$, where

$$
\begin{aligned}
t & =\frac{\prod_{i=1}^{m} a_{i}^{m_{i}}}{\prod_{i=m+1}^{N} a_{i}^{m_{i}}}, \\
m^{\prime} & =\sum_{i=1}^{m} m_{i}
\end{aligned}
$$


and

$$
n^{\prime}=\sum_{i=m+1}^{N} m_{i}
$$

For a definition of the Meijer $G$-function see again [3] and [4].

\section{III. $H$ Function TeChNiQue}

The results in [1] and [2] can be obtained as particular cases of what is known as the $H$-Function Technique, see [5] and [6]. Suppose $X_{i}, i=1,2, \ldots, n$ are independent non-negative random variables with the pdfs $f_{X_{i}}(\cdot)$ expressed as

$$
f_{X_{i}}(x)=k_{i} H_{p_{i}, q_{i}}^{m_{i}, n_{i}}\left(c_{i} x_{i} \mid \begin{array}{c}
\left(a_{i 1}, \alpha_{i 1}\right), \ldots,\left(a_{i p_{i}}, \alpha_{i p_{i}}\right) \\
\left(b_{i 1}, \beta_{i 1}\right), \ldots,\left(b_{i q_{i}}, \beta_{i q_{i}}\right)
\end{array}\right)
$$

for $x>0$. Almost all of the non-negative distributions (including the gamma, Weibull, Maxwell, beta, half-normal, exponential, chi-square, Rayleigh, general hypergeometric and the half-Cauchy distributions) can have their pdfs expressed in the form of (4). Carter and Springer [6] showed that the pdf of the product

$$
Y=\prod_{i=1}^{n} X_{i}
$$

also takes the form of (4) with

$$
\begin{aligned}
f_{Y}(y)=\left(\prod_{i=1}^{n} k_{i}\right) H_{p_{1}+\cdots+p_{n}, q_{1}+\cdots+q_{n}}^{m_{1}+\cdots+m_{n}, n_{1}+\cdots+n_{n}} \\
\times\left(y \prod_{i=1}^{n} c_{i} \mid \begin{array}{l}
\left(\begin{array}{l}
\left.a_{11}, \alpha_{11}\right), \ldots,\left(a_{n p_{n}}, \alpha_{n p_{n}}\right) \\
\left(b_{11}, \beta_{11}\right), \ldots,\left(b_{n q_{n}}, \beta_{n q_{n}}\right)
\end{array}\right)
\end{array}\right.
\end{aligned}
$$

for $y>0$. The formula in (5) provides the $H$ technique. It can be used to derive the product of a large class of random variables, including those distributions mentioned above and those discussed in [1] and [2].

\section{DISCUSSION}

The authors feel the references and the results mentioned above can be of help to the general electrical and communication engineering community (in particular, to the readers and authors of the IEEE Transactions on antennas and Propagation, IEEE TRANSACTIONS ON COMMUNICATIONS, IEEE TRANSACTIONS ON Vehicular TeChNOLOGy, AND IEEE TRANSACTIONS ON Wireless COMMUNICATIONS). It will also help to prevent similar shortcomings in the future.

\section{REFERENCES}

[1] J. Salo, H. M. El-Sallabi, and P. Vainikainen, "The distribution of the product of independent Rayleigh random variables," IEEE Trans. Antennas Propag., vol. 54, pp. 639-643, Feb. 2006.

[2] A. M. Mathai, "Products and ratios of generalized gamma variates," Skand. Aktuarietidskr, pp. 193-198, 1972.

[3] A. M. Mathai, A Handbook of Generalized Special Functions for Statistical and Physical Sciences. Oxford, U.K.: Oxford Univ. Press, 1993.

[4] A. M. Mathai and R. K. Saxena, The H-Function With Applications in Statistics and Other Disciplines. New York: Wiley, 1978.

[5] M. D. Springer, The Algebra of Random Variables. New York: Wiley, 1979.

[6] B. D. Carter and M. D. Springer, "The distribution of products, quotients and powers of independent $H$-function variates," SIAM J. Appl. Math., vol. 33, pp. 542-558, 1977.

\section{Reply to "Comments on 'On the Distribution of the Product of Independent Rayleigh Random Variables",}

Jari Salo, Hassan M. El-Sallabi, and Pertti Vainikainen

\section{INTRODUCTION}

Having read the interesting comments by Nadarajah and Kotz, we find ourselves honored that they have dedicated time to comment on our recent paper [1]. While their comments appear to be partly directed to-and indeed very useful for-the wireless communications community at large, we shall in this reply address some of the points they have raised.

\section{REPLY TO NADARAJAH AND KotZ'S COMMENTS}

The main point of Nadarajah and Kotz is that "the result on the pdf of $Y$ given in [1] has been known before." This is partly correct. To be exact, the Meijer $G$-function form of the probability density function (pdf) of the product of independent Rayleigh variates given in [1, eq. (8)] is a special case of [2, eq. (2.6)] and [3, Theorem 4.1]. However, we hasten to add that the remaining results in [1], including the Meijer $G$-function form of the cumulative distribution function (cdf) in (9) as well as the series forms of the pdf and cdf in (10)-(15) have not, to our knowledge, appeared elsewhere (but see comments on recent literature, below). Furthermore, in addition to the distribution functions mentioned, we believe that [1] also sheds light on a number of other issues of engineering interest related to $n$-Rayleigh radio channels, including their dynamic range and measurement data analysis. In conclusion, the overlap between our paper and the references pointed out by Nadarajah and Kotz can be considered small, essentially a single equation. Nevertheless, we agree with Nadarajah and Kotz in that the bibliography of our paper would have been more complete had we been able to excavate [2] and [3]. In our opinion, however, the Scandinavian Actuary Journal [2] in particular cannot be considered a well-known statistics periodical.

Nadarajah and Kotz describe the " $H$-function technique" as a general method for deriving product distributions. We wish to point out that the $H$-function technique is essentially identical to what we call the "Mellin transform technique" in [1], where we emphasize in no less than three occasions (in the Abstract, and Sections I and III) that this is a well-known technique from statistics and accordingly cite two books by Professor Mathai where the basic idea is given.

\section{DISCUSSION}

There is a growing interest in product distributions in wireless communications research and, after the submission of [1] in April 2004, some of its results have been independently discovered and extended by other researchers. In particular, the Meijer $G$-function forms of the pdf and cdf of the product of independent Rayleigh variates have also been derived in [4] in context of approximating the distribution of a sum of Rayleigh variates. The distributions of the products of independent Nakagami- $m$, gamma, and generalized gamma variates have been considered in [5]-[7], respectively. References [2] and [3] pointed out by Nadarajah and Kotz provide very general results

Manuscript received August 9, 2006.

J. Salo was with the SMARAD Centre of Excellence, Radio Laboratory, Helsinki University of Technology, FI-02015-TKK, Finland.. He is now with European Communications Engineering, FIN-02150 Espoo, Finland (e-mail: jari.salo@eceltd.com).

H. El-Sallabi and P. Vainikainen are with the SMARAD Centre of Excellence, Radio Laboratory, Helsinki University of Technology, FI-02015-TKK, Finland (e-mail: hsallabi@cc.hut.fi; pertti.vainikainen@tkk.fi).

Digital Object Identifier 10.1109/TAP.2006.884317 